\title{
39. Postoperative Management by Prolonged Hypothermia in Brain Operation
}

\author{
- The third report-
}

\author{
Kenzo Matsuoka, Tohoru Uozumi, Muneo Kajimura, Mitsumasa Kano \\ Tsukasa Kishida, Kazuyasu NaKao, Kazuhiko Nonaka \\ Hideyuki Tanaka, Takashi Fukuhara, Yuji Hamanaka \\ Tetsuto TAKaO, and Maso Watanabe \\ First Department of Surgery, Osaka University Medical School
}

It is generally recoggnized that hypothermia is effective in preventing cerebral edema or swelling in neurosurgery.

Hypotention under hypothermia has been suggested to be one of the causative factors lowerling the intracranial pressure.

Since 1961, 131 cases of intracranial tumors, aneurysms and severe head injuries have been operated on under moderate hypothermia by surface cooling $\left(28^{\circ} \mathrm{c}\right.$ to $\left.30^{\circ} \mathrm{c}\right)$.

During the postoperative prolonged hypothermia by surface cooling, the intracranial pressure in ventricular drainage increased gradually with the increase of body temperature but not of blood pressure.

In the group under hypothermia, the time to arrive at the highest value of cerebrospinal fluid pressure was more prolonged than in the groupe under normothermia.

It is suggested in this study that hypothermia represses the increase of cerebro spinal fluid pressure after operation.

We consider that the prolonged postoperative hypothermia is effective in severe neurosurgical cases. 